\title{
Local-density-functional calculations for defect interactions in Al
}

\author{
T. Hoshino \\ Faculty of Engineering, Shizuoka University, Hamamatsu 432, Japan \\ R. Zeller and P. H. Dederichs \\ Institute für Festkörperforschung, Forschungszentrum Jülich, D-52545 Jülich, Germany
}

(Received 8 December 1995)

\begin{abstract}
We present systematic $a b$ initio local-density-functional calculations for defect interactions in Al. In particular, we calculate interaction energies of both impurity-vacancy and impurity-impurity pairs on first- and second-neighbor sites; as impurities, we choose Sc-As ( $3 d$ and $4 s p$ series) and Y-Sb ( $4 d$ and $5 s p$ series). The present method is based on the Kohn-Korringa-Rostoker Green's-function method. The calculations reproduce well the experimental values for the vacancy-solute interaction and elucidate the micromechanisms of the vacancy-impurity and impurity-impurity binding energies.
\end{abstract}

In the last decades the interactions of point defects in solids have been the subject of numerous experimental studies because knowledge of the interaction is indispensable for the understanding of many basic physical processes, such as diffusion, short-range order, segregation, ordering, etc. For instance, it is obvious that the diffusion properties of impurities in metals depend strongly on the vacancy-solute interaction energies. ${ }^{1}$ The elucidation of the micromechanism for the interatomic interactions in alloys is also important to design technologically useful alloys with good mechanical properties. ${ }^{2}$ Especially, alloys of $\mathrm{Al}$ with transition metals have received considerable attention in the material-science community because of their superior technological properties, such as light weight and high strength. More recently, the discovery of quasicrystal phases of Al-transition-metal alloys has also requested realistic calculations for the electronic structure. Theoretically many simple calculations based on the jellium model or the pseudopotential approach with linear screening theory were used to investigate the electronic structure of a vacancy in Al. However, it was reviewed by Evans and Finnis ${ }^{3}$ that these methods predict unphysical values for the vacancy formation energy. Recently various first-principles calculations based on the pseudopotential method have been carried out for the vacancy formation energy in $\mathrm{Al} .{ }^{4-10}$ For example, Chetty et al. ${ }^{10}$ succeeded in reproducing very well the experimental value of the vacancy formation energy in Al. They used the supercell approach for an isolated vacancy. It was shown that adequate sampling of the Brillouin zone is essential for the metallic systems, even for systems containing more than 100 atoms per unit cell. However, it seems to be still difficult to apply the same pseudopotential approach to the vacancy formation energies in the transition metals and to the interaction energies in alloys of $\mathrm{Al}$ with transition metals. To our knowledge, any calculations for the interaction energies of impurities in Al have up to now not been performed by $a b$ initio methods. This is the aim of the present paper.

In the past, we have successfully calculated solution energies of impurities from first principles. The calculations are based on local-density-functional theory and apply the Korringa-Kohn-Rostoker (KKR) Green's-function method for impurities ${ }^{11}$ together with a recently developed accurate total-energy formalism; ${ }^{12}$ the method is entirely free from both the pseudopotential and the supercell approximation. In the present work we use the spherical-potential approximation for the Kohn-Sham equation, but the total energy is calculated by use of the full nonspherical charge density. Based on this method, we have already succeeded in calculating vacancy-solute interaction energies in $\mathrm{Cu}, \mathrm{Ni}, \mathrm{Ag}$, and $\mathrm{Pd}$. The experimentally known interaction energies of vacancy solutes in $\mathrm{Cu}, \mathrm{Ni}, \mathrm{Ag}$, and $\mathrm{Pd}$ are very well reproduced by the calculations and the microscopic mechanisms have been elucidated. ${ }^{13,14}$ Further the calculations have been applied to the interaction energies of the impurities with the probe atoms $\left({ }^{99} \mathrm{Rh},{ }^{100} \mathrm{Pd},{ }^{111} \mathrm{In}\right)$ in $\mathrm{Ag}$ and $\mathrm{Pd},{ }^{15}$ as well as the impurity-impurity interactions in $\mathrm{Cu}, \mathrm{Ni}, \mathrm{Ag}$, and $\mathrm{Pd} .{ }^{16}$

In this paper, we use the same method to study the interaction energies of defects in Al. In particular we calculate the interaction energies of both vacancy-impurity and impurityimpurity (of the same kind) pairs by considering both nearest-neighbor and the next-nearest-neighbor configurations. Since at the present stage we cannot treat the lattice relaxation around the impurities, we consider only the impurity series Sc-As $(3 d-4 s p)$ and Y-Sb $(4 d-5 s p)$, the WignerSeitz radii of which are not too different from that of Al.The present systematic studies give reliable information to build a global picture of the bonding properties for $\mathrm{Al}$ alloys with transition-metal elements. We apply density-functional theory in the local density approximation of von Barth and Hedin with the parameters as given by Morruzi, Janak, and Williams. $^{17}$ In the KKR Green's-function method, the Green's function of the system is expanded in each cell into radial eigenfunctions of the local potential within the circumscribing sphere of the Wiger-Seitz cell. All multiplescattering information is contained in the structural Green'sfunction matrix $G_{L L^{\prime}}^{n n^{\prime}}(E)$, which is related to $\stackrel{\circ}{G}_{L L^{\prime}}^{n n^{\prime}}(E)$ of the ideal crystal by a Dyson equation

$$
G_{L L^{\prime}}^{n n^{\prime}}(E)=\stackrel{\circ}{G}_{L L^{\prime}}^{n n^{\prime}}(E)+\sum_{n^{\prime \prime}, L^{\prime \prime}} \stackrel{\circ}{G}_{L L^{\prime \prime}}^{n n^{\prime \prime}}(E) \Delta t_{L^{\prime \prime}}^{n^{\prime \prime}}(E) G_{L^{\prime \prime} L^{\prime}}^{n^{\prime \prime} n^{\prime}}(E),
$$




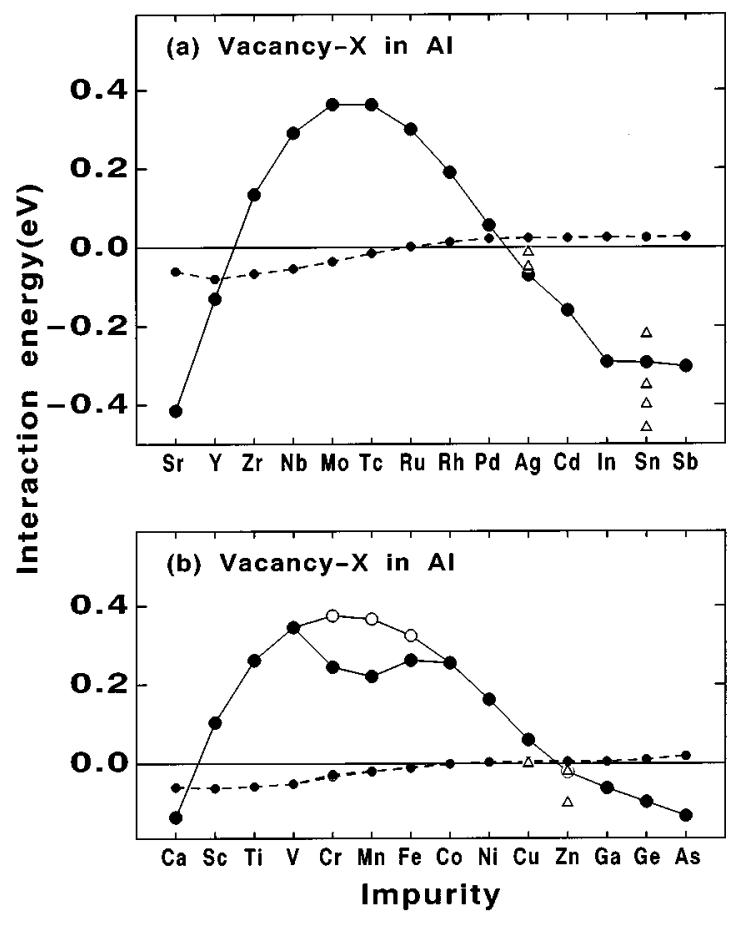

FIG. 1. Interaction energies (-) of (a) $4 d$ and $5 s p$ impurities as well as (b) $3 d$ and $4 s p$ impurities with a vacancy in Al. The large solid circles $(-)$ indicate the interaction energies for the nearestneighbor sites, and the small solid circles $(\bullet)$ refer to the nextnearest-neighbor sites. Open circles $(O, \circ)$ refer to calculations without spin polarization. The measured values $(\triangle)$ are also shown.

where $\Delta t_{L^{\prime \prime}}^{n^{\prime \prime}}(E)=t_{L^{\prime \prime}}^{n^{\prime \prime}}(E)-{\stackrel{\circ}{t^{\prime \prime}}}_{L^{\prime \prime}}^{\prime \prime}(E)$ is the difference from the host $t$ matrix ${\stackrel{\circ}{t^{\prime \prime}} n^{\prime \prime}}^{\prime \prime}(E)$. For the present impurity system, we calculate self-consistently all potentials at the defect sites and at the nearest-neighbor sites to at least one of the defects. This amounts up to 20 potentials for the nearest-neighbor configuration and 22 for the next nearest-neighbor one (see Fig. 1 in Ref. 16). The maximum angular momentum $l_{\max }$ for the Green's function is chosen to be 3 . It was shown in Ref. 12 that both approximations are sufficient to obtain reliable total energies. The energy integration is performed by a contour integral in the complex energy plane. ${ }^{18}$ The doublecounting contributions for both the Coulomb and the exchange energies are calculated by use of the full anisotropic charge density in each Wigner-Seitz cell. The integrations over the exact faceted Wigner-Seitz cell can be performed by introducing a shape function, i.e., a Heaviside function being equal to 1 inside and 0 outside the cell, which is expanded into spherical harmonics within the circumscirbing sphere of the Wigner-Seitz cell. ${ }^{14,19}$ However, in order to solve the Kohn-Sham equation, we used only the spherical parts ( $l$ $=0$ ) of the full anisotropic potentials, which are obtained by use of the full anisotropic charge densities. For the details the readers are referred to Ref. 14.

First, we will discuss the nearest-neighbor interaction energies of impurities with a vacancy; the interaction energy is related to the difference between the host-impurity and hosthost bond energies since by the neighboring of an impurity to the vacancy a broken host-host bond is replaced by a broken host-impurity bond. Figure 1(a) shows the calculated results

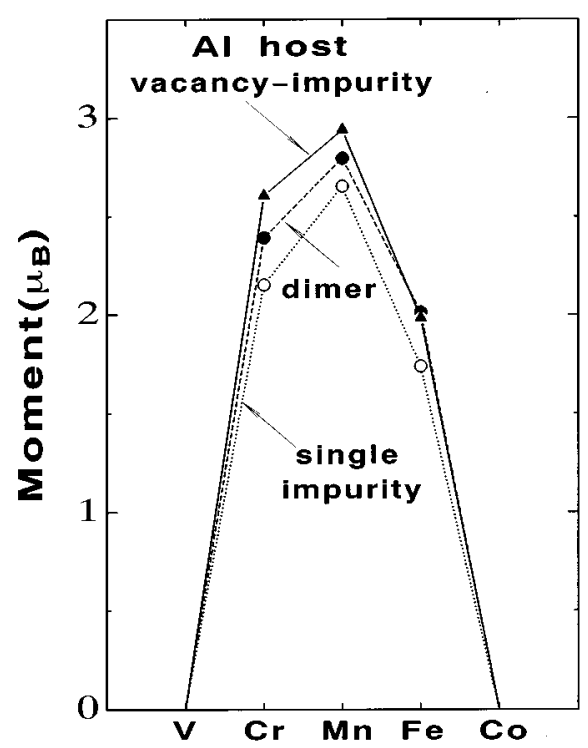

FIG. 2. Local moments of single impurities (dotted line), vacancy-impurity pairs (solid line), and dimers (dashed line) in Al.

for $4 d$ and $5 s p$ impurities. Positive energies mean a repulsion between the vacancy and the impurity; negative energies mean attraction. For $4 d$ impurities, except at the beginning of the transition-metal series, one can see a strong repulsion in the form of a parabolic behavior with a maximum in the middle of the transition-metal series. Contrary to this, for $5 s p$ impurities the interaction is attractive; the magnitude changes linearly from $\mathrm{Ag}$ to In and is more or less constant from In to $\mathrm{Sb}$. These results can qualitatively be explained by comparing the strengths of the host-impurity and host-host bonds. The strong repulsion can be understood by the break up of one strong $s p$-d (host-impurity) bond, which is not overcome by the energy gain due to the formation of the $s p-s p$ (host-host) bond. On the other hand, the attractive tendency around $s p$ impurities might be understood by the energy gain due to the formation of $s p-s p$ (host-host) bond, which is stronger than the $s p-s p$ (host-impurity) bond. The

TABLE I. Local magnetic moments for three different nearestneighbor configurations of $3 d$ impurities in $\mathrm{Al}$, i.e., (a) single impurities, (b) single impurities adjacent to a vacancy, and (c) impurity dimers. For (b) and (c) the values for the second-neighbor configurations as well as for the first-neighbor configurations are shown. It is clearly seen that the local magnetic moment increases by the neighboring of a vacancy and also by pairing of $3 d$ impurities. For the dimers the values for the magnetic configuration $(F$, ferromagnetic; $\mathrm{AF}$, antiferromagnetic) of the ground state are given.

\begin{tabular}{llll}
\hline \hline Impurity & \multicolumn{1}{c}{$\mathrm{Cr}$} & $\mathrm{Mn}$ & $\mathrm{Fe}$ \\
\hline $\begin{array}{l}\text { (a) Single impurities } \\
\text { (b) Single impurities with }\end{array}$ & 2.153 & 2.653 & 1.740 \\
$\quad$ & & & \\
$\quad$ a vacancy & & & \\
$\quad$ first-neighbor & 2.605 & 2.941 & 1.983 \\
$\quad$ second-neighbor & 2.131 & 2.649 & 1.746 \\
(c) Impurity dimers & & & \\
$\quad$ first-neighbor & $2.393(\mathrm{AF})$ & $2.796(\mathrm{~F})$ & $2.013(\mathrm{~F})$ \\
$\quad$ second-neighbor & $2.322(\mathrm{~F})$ & $2.731(\mathrm{~F})$ & $1.780(\mathrm{AF})$ \\
\hline \hline
\end{tabular}


interaction energies of $3 d$ and $4 s p$ impurities with a vacancy, shown in Fig. 1(b), can also be explained by the same mechanism. However, it should be noted that the dip in the middle in $3 d$ series, shown in Fig. 1(b) [non-spin-polarized $(\bigcirc)$ and spin-polarized $(\bigcirc)$ calculations], arises from magnetism: The reduction of attraction seen in the spin-polarized calculations (0) is due to the energy gain obtained by the increase of local impurity moments, shown in Fig. 2 and Table I, caused by the neighboring of a vacancy; large magnetic effects like this one are well known from surface magnetism. The detailed discussion is given elsewhere. ${ }^{20}$

Here we will give a brief comparison with experimental results. From the measurement of equilibrium vacancy concentration in AlAg alloys, Beaman et al. ${ }^{21}$ obtained a value of $-0.05 \mathrm{eV}$ for the interaction of a $\mathrm{Ag}$ impurity with a vacancy in $\mathrm{Al}$, whereas Rivieré and Grilhé ${ }^{22}$ obtained -0.01 $\mathrm{eV}$. Both values compare well with our calculated value $-0.07 \mathrm{eV}$. For Sn, many experiments were carried out with values distributing between -0.22 and $-0.40 \mathrm{eV}^{23}$ According to a Mössbauer experiment, ${ }^{23}$ the magnitude of the binding energy is estimated to be less than $0.30 \mathrm{eV}$. Thus, within the experimental uncertainties, all values are compatible with our value $-0.29 \mathrm{eV}$. Moreover, an experimental value of 0.0 $\mathrm{eV}$ (equilibrium vacancy concentration) has been reported for $\mathrm{Cu},{ }^{24}$ and -0.02 and $-0.10 \mathrm{eV}$ (quenching technique) for $\mathrm{Zn} ;{ }^{1,25}$ these values compare well with our values, $0.04 \mathrm{eV}$ for $\mathrm{Cu}$ and $-0.02 \mathrm{eV}$ for $\mathrm{Zn}$.

It is interesting to compare our results with experimental $\Delta Q$ data $^{26,27}$ from impurity diffusion in $\mathrm{Al}$ giving the difference of the impurity activation energies with respect to the host value. Whereas in the noble metals the $\Delta Q$ values are very well given by the calculated binding energies to the vacancy, this is apparently not the case in Al. Here, in qualitative agreement with our results, positive $\Delta Q$ values, signalizing repulsion, are reported for transition-metal impurities and negative values, meaning attraction, for the $s p$ impurities. However, at least for the transition-metal impurities, the absolute $\Delta Q$ values are much larger than the calculated binding energies. For instance, for $\mathrm{Zr}$, Mo, and $\mathrm{Cr}$ the reported $\Delta Q$ values are larger than $1 \mathrm{eV}$, thus much larger than the binding energies in Figs. 1(a) and 1(b). This is presumably due to the fact that in Al the saddle-point energy for diffusion is strongly increased for the transition-metal impurities, which apparently plays a minor role in the noble metals, where the binding energies dominate the diffusion behavior of the impurities. ${ }^{27}$

Figures 1(a) and 1(b) include also the interaction energies for the next-nearest-neighbor configuration. As a general rule and in agreement with earlier results for noble metals, ${ }^{16}$ these interactions are usually much smaller than the nearestneighbor ones. This is clearly the case for the present systems. We obtain a very weak and more or less constant repulsion for the $s p$ elements of both series and a slight attraction for the transition-metal elements at the beginning and in the middle of the $d$ series. It is also found that the magnetic interaction of $3 d$ impurities [Fig. 1(b)] is very small because of very small change of magnetic moments due to a vacancy on second neighbor site, shown in Table I.

Next, we discuss the nearest-neighbor interaction energies of impurity-impurity pairs; the interaction energy is related to the creation of two bonds, i.e., one impurity-impurity bond

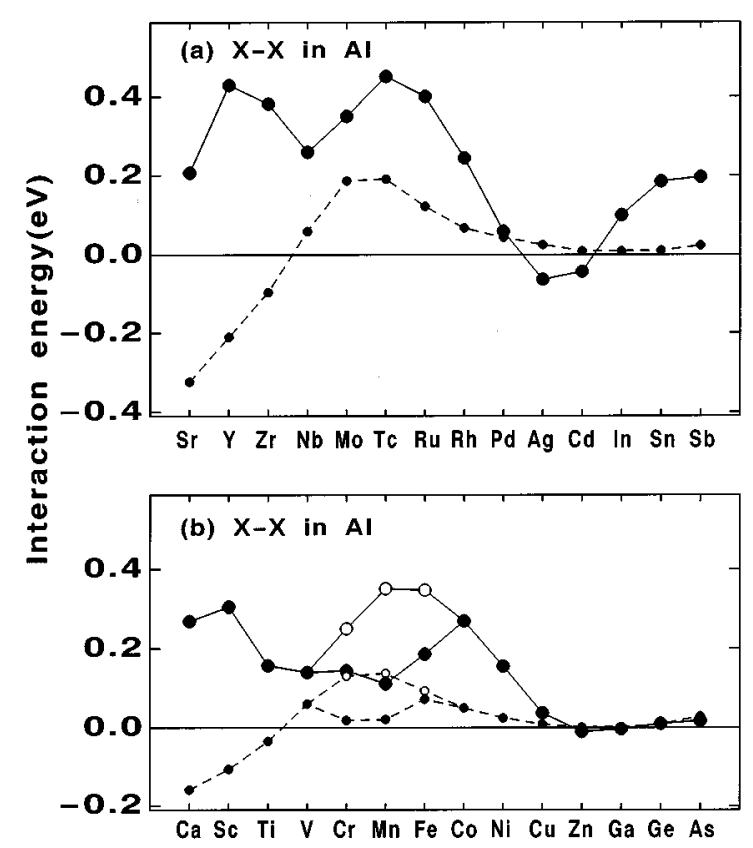

FIG. 3. Interaction energies of impurity-impurity pairs both of (a) $4 d$ and $5 s p$ elements and (b) $3 d$ and $4 s p$ elements in Al.The large solid circles $(-)$ indicate the interaction energies for the nearest-neighbor sites, and the small solid circles $(\bullet)$ refer to the next-nearest-neighbor sites. Open circles $(\bigcirc, \circ)$ refer to calculations without spin polarization.

and one host-host bond, and the breakup of two hostimpurity bonds. Figure 3(a) shows the calculated results for $4 d$ and $5 s p$ impurities. We find strong repulsion for $4 d$ impurities, while the interaction is weakly attractive or repulsive for $5 s p$ impurities: weak attraction for $\mathrm{Ag}$ and $\mathrm{Cd}$ and weak repulsion for $\mathrm{In}, \mathrm{Sn}$, and $\mathrm{Sb}$. The strong repulsion in the $4 d$ series is mainly due to the breakup of two strong $s p$ - $d$ (host-impurity) bonds. It is also noted that the four $s p$ - $d$ (host-impurity) bonds between the impurity atoms and the host atoms neighboring to both the impurities [bonds (1)-(2) shown in Fig. 1(a) in Ref. 16] are also weaker. The dip found in the middle of $4 d$ series can be explained by the energy gain due to the formation of one strong $d-d$ bond. These calculated results, corresponding to dilute limit of alloys, for the $4 d$ series qualitatively agree with those obtained by Carlsson, ${ }^{28}$ who used supercell calculations for the ordered alloys, and also with the observed tendency of the strong ordering., 28 Carlsson explained the dip ("two maxima") by using two kinds of moments (second moments and fourth moments); ${ }^{28}$ the second moment corresponds to the formation of the $d$ - $d$ (impurity-impurity) bond, while the fourth moment is necessary to describe the breakup of the $s p-d$ (host-impurity) bond. The relatively weak interaction for In, Sn, and Sb can also be explained by considering the similar character of $s p$ impurities with the host atom; the similarity of these elements can be seen in Miedema's parameters, ${ }^{2}$ leading to almost zero for the solution energies of $5 s p$ impurities in $\mathrm{Al}$. For $\mathrm{Ag}$ and $\mathrm{Cd}$, the energy gain due to the formation of $s p$ - $s p$ (host-host) bond may overcome the energy loss due to the weak $s p$-sp (host-impurity) bonds; hence, the impurity-impurity interaction is attractive.

The repulsive interaction of $3 d$ impurities can be under- 
stood along the same line as the one of their $4 d$ counterparts. Because of the stronger localization of $3 d$ orbitals, resulting in a weaker hybridization, the interactions are, however, somewhat smaller. In addition, magnetic effects are important for $\mathrm{Cr}, \mathrm{Mn}$, and Fe impurities, lowering the repulsion. This unusual behavior, being opposite to the one found in noble metals, ${ }^{16}$ can be traced back to the behavior of the local moments, which, contrary to the behavior in the noble metals, increase in the dimer configuration (Fig. 2), resulting in an energy gain due to spin polarization. This increase is due to the fact that for the pair configuration the hybridization of the $3 d$ electrons is reduced compared to the isolated impurities, which clearly dominates the behavior. The interaction of $4 s p-4 s p$ is also similar to $5 s p-5 s p$, but weaker as a result of weaker hybridization.

Note that the repulsive behavior for the transition-metal pairs in $\mathrm{Al}$ is very different from the attractive behavior found in the nobel metals. ${ }^{16}$ In nobel metals, because of the much weaker hybridization with the host, the $s p$-d (hostimpurity) bond is very weak and the interaction is attractive due to the formation of the much stronger $d$ - $d$ (impurityimpurity) bond. In Al the strength of the bonds is reversed and the behavior is determined by the dominating $s p-d$ (host-impurity) bond. These characteristic features are essential for the desirable technological properties of alloys of $\mathrm{Al}$ with transition-metal elements.

Figures 3(a) and 3(b) also include the interaction energies for the next-nearest-neighbor configuration. As discussed before, these interactions are generally weaker than the nearestneighbor ones, but are still large compared with those of vacancy-impurity pairs. The repulsive behavior around the transition-metal impurities may be understood by considering the weakening of the four $s p$ - $d$ [host (2)-impurity (1)] bonds, shown in Fig. 1(b) in Ref. 16. The attraction around early transition-metal impurities may be explained by the bonding of rather delocalized $d$ orbitals and becomes large toward $\mathrm{Sr}(4 d)$ [Fig. 3(a)] and $\mathrm{Ca}(3 d)$ [Fig. 3(b)]. The attractive magnetic interaction of $3 d$ impurities is due to the increase of magnetic moments, caused by pairing of impurities at the second-neighbor sites, shown in Table I.

In summary, we have shown that the present method reproduces available experimental results of interaction energies of vacancy-impurity pairs in Al. Based on the calculated results, we also discussed the micromechanism of interaction energies of vacancy-impurity $(3 d-4 s p, 4 d-5 s p)$ and impurity-impurity pairs in Al. The repulsive interaction of a vacancy with $3 d$ and $4 d$ impurities can be understood by the breakup of the strong $s p$ - $d$ (host-impurity) bonds. The repulsive interaction of the $3 d$ and $4 d$ impurity pairs is also explained by the breakup of the strong $s p$ - $d$ (host-impurity) bonds and is very different from the attractive pair interaction of $3 d$ and $4 d$ impurities in nobel metals $\mathrm{Cu}$ and $\mathrm{Ag}$. ${ }^{16}$

The main part of the present results were obtained with the computers at Forschungszentrum Jülich. This work was partly supported by a Grand-in-Aid for Scientific Research on Priority Areas, "Computational Physics as a New Frontier in Condensed Matter Research," from the Ministry of Education, Science and Culture.
${ }^{1}$ For a review, see M. Doyama, J. Nucl. Mater. 69\&70, 350 (1978); in Diffusion in Solids Unresolved Problems, edited by G. E. Murch (Trans Tech Publications, Zürich, 1992).

${ }^{2}$ F. R. de Boer, R. Boom, W. C. Mattens, A. Miedema, and A. K. Niessen, in Cohesion in Metals, edited by F. R. de Boer, and D. G. Pettifor (North-Holland, Amsterdam, 1985), Vol. I.

${ }^{3}$ R. Evans and M. W. Finnis, J. Phys. F 6, 843 (1976).

${ }^{4}$ M. J. Gillan, J. Phys. Condens. Matter 1, 689 (1989).

${ }^{5}$ R. W. Jansen and B. M. Klein, J. Phys. Condens. Matter 1, 8359 (1989).

${ }^{6}$ A. De. Vita and M. J. Gillan, J. Phys. Condens. Matter 3, 6225 (1991)

${ }^{7}$ R. Benedek, L. H. Yang, C. Woodward, and B. I. Min, Phys. Rev. B 45, 2607 (1992).

${ }^{8}$ A. Seeger and M. Fähnle, Computational Aided Innovation of New Material (North-Holland, Amsterdam, 1993), Vol. 2, p. 439.

${ }^{9}$ M. W. Finnis, J. Phys. Condense. Matter 2, 331 (1990).

${ }^{10}$ N. Chetty, M. Weinert, T. S. Rahman, and J. W. Davenport, Phys. Rev. B 52, 6313 (1995).

${ }^{11}$ P. J. Braspenning, R. Zeller, A. Lodder, and P. H. Dederichs, Phys. Rev. B 29, 703 (1984).

${ }^{12}$ B. Drittler, M. Weinert, R. Zeller, and P. H. Dederichs, Phys. Rev. B 39, 930 (1989).

${ }^{13}$ U. Klemradt, B. Drittler, R. Zeller, and P. H. Dederichs, Phys. Rev. Lett. 64, 2803 (1991).

${ }^{14}$ U. Klemradt, B. Drittler, T. Hoshino, R. Zeller, P. H. Dederichs, and N. Stefanou, Phys. Rev. B 43, 9487 (1991).

${ }^{15}$ T. Hoshino, B. Drittler, R. Zeller, and P. H. Dederichs, Phys. Rev.
B 45, 12202 (1992); T. Hoshino, A. Shimizu, R. Zeller, and P. H. Dederichs, ibid. 53, 5247 (1996).

${ }^{16}$ T. Hoshino, W. Schweika, R. Zeller, and P. H. Dederichs, Phys. Rev. B 47, 5106 (1993).

${ }^{17}$ V. R. Moruzzi, J. F. Janak, and A. R. Williams, Calculated Electronic Properties of Metals (Pergamon, New York, 1978).

${ }^{18}$ R. Zeller, J. Deutz, and P. H. Dederichs, Solid State Commun. 44, 993 (1982).

${ }^{19}$ U. Klemradt, Diploma thesis, Technical University Aachen, Aachen, Germany, 1989.

${ }^{20}$ T. Hoshino, R. Zeller, P. H. Dederichs, and M. Weinert, Europhys. Lett. 24, 495 (1993); T. Hoshino, R. Zeller, and P. H. Dederichs, J. Magn. Magn. Mater. 140-144, 39 (1995).

${ }^{21}$ D. R. Beaman, R. W. Balluffi, and R. O. Simmons, Phys. Rev. 134, A532 (1964).

${ }^{22}$ J. P. Rivieré and J. Grilhé, Phys. Status Solidi A 5, 701 (1971).

${ }^{23}$ See, e.g., K. O. Sorensen and R. M. J. Cotterill, Acta Metall. 22, 1331 (1974).

${ }^{24}$ G. Rivaud, J. P. Rivieré, and J. Grilhé; Scr. Met. 6, 65 (1972).

${ }^{25}$ See, e.g., A. D. King, K. O. Sorensen, and R. M. J. Cotterill, Acta Metall. 22, 1331 (1974).

${ }^{26}$ Diffusion in Solid Metals and Alloys, edited by H. Mehrer Landolt-Börnstein, New Series, Group III, Vol. 26 (SpringerVerlag, Berlin, 1990).

${ }^{27}$ G. Rummel, T. Zumkley, G. Erdélyi, C. Freitag, G. Hood, and H. Mehrer, Defect Diffusion Forum 95-98, 715 (1993); G. Rummel, T. Zumkley, M. Eggersmann, K. Freitag, and H. Mehrer, Z. Metallk. 86, 122 (1995); 86, 131 (1995).

${ }^{28}$ A. E. Carlsson, Phys. Rev. B 40, 912 (1989). 